$9-1-2011$

\title{
The Eigenvalues of a Tridiagonal Matrix in Biogeography
}

Boris Igelnik

BMI Research

Daniel J. Simon

Cleveland State University, d.j.simon@csuohio.edu

Follow this and additional works at: https://engagedscholarship.csuohio.edu/enece_facpub

Part of the Algebra Commons, and the Electrical and Computer Engineering Commons How does access to this work benefit you? Let us know!

Publisher's Statement

(c) 2011 Elsevier

\section{Original Citation}

Igelnik, B., \& Simon, D. (2011). The eigenvalues of a tridiagonal matrix in biogeography. Applied Mathematics and Computation, 218, 1, 195-201.

\section{Repository Citation}

Igelnik, Boris and Simon, Daniel J., "The Eigenvalues of a Tridiagonal Matrix in Biogeography" (2011). Electrical Engineering \& Computer Science Faculty Publications. 15.

https://engagedscholarship.csuohio.edu/enece_facpub/15

This Article is brought to you for free and open access by the Electrical Engineering \& Computer Science Department at EngagedScholarship@CSU. It has been accepted for inclusion in Electrical Engineering \& Computer Science Faculty Publications by an authorized administrator of EngagedScholarship@CSU. For more information, please contact library.es@csuohio.edu. 


\section{THE EIGENVALUES OF A TRIDIAGONAL MATRIX IN BIOGEOGRAPHY}

Boris Igelnik, BMI Research, Richmond Heights, $\mathrm{OH}$

Dan Simon, Cleveland State University, Cleveland, $\mathrm{OH}$

\section{Main result and related work}

We prove the following theorem in this paper.

Theorem 1. The $(n+1) \times(n+1)$ tridiagonal matrix

$$
A=\left[\begin{array}{ccccc}
-1 & 1 / n & 0 & \cdots & 0 \\
n / n & -1 & 2 / n & \ddots & \vdots \\
\vdots & \ddots & \ddots & \ddots & \vdots \\
\vdots & \ddots & 2 / n & -1 & n / n \\
0 & \ldots & 0 & 1 / n & -1
\end{array}\right]
$$

for any natural $n \geqslant 4$ has the $(n+1)$ eigenvalues

$$
x(A)=\{0,-2 / n,-4 / n, \ldots,-2\}=-2 k / n, \quad k \in[0, n] .
$$

This theorem was stated as a conjecture in [14] and is proven here in Sections 2-4. This type of matrix arises in biogeography theory, and its application is discussed in Section 5.

There have been many papers published on explicit solutions of eigensystems of tridiagonal matrices of different structures. Some research on this topic includes $[2,5,7,10,11,16]$. The common feature of $[2,5,7,16]$ is that the tridiagonal matrix $A$ has the following structure: 


$$
A=\left[\begin{array}{cccccc}
-\alpha+b & c & 0 & 0 & \cdots & 0 \\
a & b & c & 0 & \ddots & 0 \\
0 & a & b & c & \ddots & 0 \\
\vdots & \ddots & \ddots & \ddots & \ddots & \vdots \\
0 & 0 & 0 & a & b & c \\
0 & 0 & 0 & 0 & a & -\beta+b
\end{array}\right] .
$$

That is, the subdiagonals are equal, and the superdiagonals are equal. However, this is not true in the matrix that we consider in Eq. (1).

In $[10,11]$ the subdiagonals and superdiagonals may have different entries $\left\{c_{1}, c_{2}, \ldots, c_{n}\right\}$ and $\left\{a_{1}, a_{2}, \ldots, a_{n}\right\}$ respectively, but these entries satisfy the condition

$$
a_{j} c_{j}=\left\{\begin{array}{l}
d_{1}^{2} \text { if } j \text { is odd, } \\
d_{2}^{2} \text { if } j \text { is even, }
\end{array} \text { for } j \in[1, n],\right.
$$

where $d_{1}$ and $d_{2}$ are, generally speaking, complex numbers. It is easy to see that this condition is not satisfied in Eq. (1). Results in $[10,11]$ differ from each other in that the latter allows a more general structure for the main diagonal than the former.

There are a number of additional references related to the explicit solution of eigensystems for tridiagonal matrices of specific structures $[1,3,4,6,13,15]$. However, the tridiagonal matrices considered in those papers have structures either with equal entries in each off-diagonal, or with off-diagonal entries $\left\{c_{1}, c_{2}, \ldots, c_{n}\right\}$ and $\left\{a_{1}, a_{2}, \ldots, a_{n}\right\}$ satisfying the conditions of $[10,11]$.

We see that our result, Theorem 1 , considers a different matrix structure than previously published work. Section 2 transforms the eigensystem equation of the matrix $A$ in Eq. (1) to a form that is amenable to the proof of Theorem 1. Section 3 proves Theorem 1 for the zero eigenvalue, and Section 4 proves the theorem for the nonzero eigenvalues. Section 5 discusses the application of the theorem to biogeography.

\section{Transformation of the eigensystem equation}

The $(n+1)$ eigensystem equations for $A$ can be written as follows:

$$
\begin{aligned}
& -V_{0}(x)+\frac{1}{n} V_{1}(x)=x V_{0}(x) \\
& \frac{n}{n} V_{0}(x)-V_{1}(x)+\frac{2}{n} V_{2}(x)=x V_{1}(x) \\
& \frac{n-1}{n} V_{1}(x)-V_{2}(x)+\frac{3}{n} V_{3}(x)=x V_{2}(x) \\
& \vdots \\
& \frac{2}{n} V_{n-2}(x)-V_{n-1}(x)+\frac{n}{n} V_{n}(x)=x V_{n-1}(x) \\
& \frac{1}{n} V_{n-1}(x)-V_{n}(x)=x V_{n}(x),
\end{aligned}
$$

where $x$ is an eigenvalue with the corresponding eigenvector $V(x)=\left[V_{0}(x) \ldots V_{n}(x)\right]^{T}$.

The following transformation of Eq. (3) plays a key role in our proof of Theorem 1. We add the first equation of Eq. (3) to the second one, obtaining a transformed second equation; we then add the new second equation to the third equation of Eq. (3), obtaining a transformed third equation. We continue this process to transform the tridiagonal system of Eq. (3) to the following upper triangular form:

$$
\begin{aligned}
& -V_{0}(x)+\frac{1}{n} V_{1}(x)=x V_{0}(x) \\
& -\frac{n-1}{n} V_{1}(x)+\frac{2}{n} V_{2}(x)=x\left[V_{0}(x)+V_{1}(x)\right] \\
& -\frac{n-2}{n} V_{2}(x)+\frac{3}{n} V_{3}(x)=x\left[V_{0}(x)+V_{1}(x)+V_{2}(x)\right] \\
& \vdots \\
& -\frac{1}{n} V_{n-1}(x)+\frac{n}{n} V_{n}(x)=x \sum_{i=0}^{n-1} V_{i}(x) \\
& 0=x \sum_{i=0}^{n} V_{i}(x) .
\end{aligned}
$$


From the last equation of Eq. (4), we obtain the following two nonintersecting cases:

$$
x=0, \text { or } x \neq 0 \text { and } \sum_{i=0}^{n} V_{i}(x)=0 .
$$

We consider the $x=0$ case in Section 3, and the $x \neq 0$ case in Section 4 .

\section{Proof of the zero eigenvalue}

Substituting $x=0$ in the first $n$ equations of Eq. (4) yields

$$
\begin{aligned}
& -V_{0}(0)+\frac{1}{n} V_{1}(0)=0 \\
& -\frac{n-1}{n} V_{1}(0)+\frac{2}{n} V_{2}(0)=0 \\
& -\frac{n-2}{n} V_{2}(0)+\frac{3}{n} V_{3}(0)=0 \\
& \vdots \\
& -\frac{1}{n} V_{n-1}(0)+\frac{n}{n} V_{n}(0)=0 .
\end{aligned}
$$

Note from Eq. (6) that we must have $V_{i}(0) \neq 0$ for all $i \in[0, n]$; otherwise $V(0)=0$, which is not a valid eigenvector. Recall that eigenvectors are not defined uniquely, but are defined only up to a nonzero scaling factor. In the following we will choose that factor so that

$$
V_{n}(0)=1=\left(\begin{array}{l}
n \\
n
\end{array}\right)
$$

Substituting this value of $V_{n}(0)$ in the last equation of Eq. (6) yields

$$
V_{n-1}(0)=n=\left(\begin{array}{c}
n \\
n-1
\end{array}\right)
$$

We substitute this value of $V_{n-1}(0)$ in the penultimate equation of Eq. (6) (i.e., the equation which contains $V_{n-1}(0)$ and $V_{n-2}(0)$, and which is not shown explicitly in Eq. (6)). We continue this process for all of the terms in Eq. (6) to derive

$$
V_{k}(0)=\left(\begin{array}{l}
n \\
k
\end{array}\right)=\frac{n !}{k !(n-k) !} \text { for } k \in[0, n]
$$

This completes the eigensystem solution for $x=0$.

\section{Proof of the nonzero eigenvalues}

Using Eq. (5), the system of Eq. (4) can be written as

$$
\begin{aligned}
& -\frac{n}{n} V_{0}(x)+\frac{1}{n} V_{1}(x)=-x \sum_{i=1}^{n} V_{i}(x) \\
& -\frac{n-1}{n} V_{1}(x)+\frac{2}{n} V_{2}(x)=-x \sum_{i=2}^{n} V_{i}(x) \\
& \vdots \\
& -\frac{1}{n} V_{n-1}(x)+\frac{n}{n} V_{n}(x)=-x \sum_{i=n}^{n} V_{i}(x)=-x V_{n}(x) \\
& \sum_{i=0}^{n} V_{i}(x)=0 .
\end{aligned}
$$

As in the preceding section, we let

$$
V_{n}(x)=1 .
$$

Theorem 1 for the nonzero eigenvalues is proven in this section by induction over arbitrary natural $n$. We first present a preliminary step in Section 4.1 that follows from the rational root theorem and that motivates our conjecture that the eigenvalues $x$ are of the form $x=-2 k / n$. Then, assuming that $x=-2 k / n$, we obtain the eigenvector for $n=6$ in Section 4.2. This forms the first step in our inductive proof; we found that the eigenvectors for $n<6$ do not provide enough structure to 
complete the inductive proof. The next step, which is mentioned at the end of Section 4.2 and which is omitted for brevity, is to prove by induction over $n$ that the eigenvector expression obtained in Section 4.2 holds for arbitrary natural $n$. The final step of the proof, which is in Section 4.3, explicitly shows that the last equation in Eq. (10) is satisfied, which completes the proof.

\subsection{Justification for the nonzero eigenvalue conjecture}

From the last equation of Eq. (10), we see that $V_{n}(x)$ is a zeroth degree polynomial. From the penultimate equation of Eq. $(10)$, we see that $V_{n-1}(x)$ is a first degree polynomial. Continuing in this manner, we see that $V_{n-i}(x)$ is an ith degree polynomial for $i \in[0, n]$. Therefore, $\sum_{i}^{n}{ }_{0} V_{i}(x)$ is an $n$th degree polynomial and the last equation of Eq. (10) can be written as

$$
\sum_{i=0}^{n} V_{i}(x)=a_{n} x^{n}+a_{n-1} x^{n-1}+\cdots+a_{1} x+a_{0}=0,
$$

where $a_{n}, a_{n-1}, \ldots, a_{0}$ are constant coefficients. This equation has $n$ roots. It is easy to calculate the coefficients $a_{n}$ and $a_{0}$. We use Eqs. (9) and (12) to obtain

$$
a_{0}=\sum_{i=0}^{n} V_{i}(0)=\sum_{i=0}^{n}\left(\begin{array}{c}
n \\
i
\end{array}\right)=\left[(1+y)^{n}\right]_{y=1}=2^{n} .
$$

Since $V_{0}(x)$ is the only $n$th degree polynomial in the sum on the left side of Eq. (12), the value of $a_{n}$ in Eq. (12) is given by the coefficient of $x^{n}$ in the polynomial $V_{0}(x)$. Using Eq. (10) to recursively solve for $V_{k}(x)$ starting with $k=n$ gives

$$
\begin{aligned}
& V_{n}(x)=1 \\
& V_{n-1}(x)=n(1+x)=n x+n \\
& V_{n-2}(x)=\frac{n}{2}\left(\frac{n-1}{n} V_{n-1}+x\left(V_{n-1}+V_{n}\right)\right)=\frac{n^{2}}{2} x^{2}+\cdots \\
& V_{n-3}(x)=\frac{n}{3}\left(\frac{n-2}{n} V_{n-2}+x\left(V_{n-2}+V_{n-1}+V_{n}\right)\right)=\frac{n^{3}}{3 !} x^{3}+\cdots \\
& \vdots \\
& V_{0}=\frac{n}{n}\left(\frac{1}{n} V_{1}+x \sum_{i=1}^{n} V_{i}\right)=\frac{n^{n}}{n !} x^{n}+\cdots
\end{aligned}
$$

We see that the coefficient of $x^{n}$ in $V_{0}(x)$ is equal to $n^{n} / n !$. Since $V_{0}(x)$ is the only $V_{k}(x)$ which includes an $x^{n}$ term, $a^{n}$ in Eq. (12) is equal to $n^{n} / n$ !. We thus multiply Eq. (12) by $n$ ! to make all of the coefficients integers. This gives us the modified coefficients $a_{n}=n^{n}$ and $a_{0}=2^{n} n !$. We see that $n$ is a divisor of $a_{n}$, and the divisors of $a_{0}$ include $2 k$ for $k \in[1, n]$. Some evidence (but not proof) for Eq. (2) comes from combining these facts with the rational root theorem:

If Eq. (12) has a rational root $x=p / q$, where $p$ and $q$ are co-prime, then $p$ is a divisor of $a_{0}$ and $q$ is a divisor of $a_{n}$.

\subsection{Eigenvectors for $x \neq 0$}

In order to find a way to prove Eq. (2) for $x \neq 0$, we will first derive $V_{n}(x), V_{n-1}(x), \ldots$, and $V_{0}(x)$, for $n=6$ and for $x=-2 k / n$, where $k \in[1, n]$. Manipulating Eq. (10) with some tedious but simple computations yields the following:

$$
\begin{aligned}
& V_{n}(x)=1 \\
& V_{n-1}(x)=n-2 k \\
& V_{n-2}(x)=\frac{(n-2 k)(n-2 k-1)}{2 !}-k \\
& V_{n-3}(x)=\frac{(n-2 k)(n-2 k-1)(n-2 k-2)}{3 !}-k(n-2 k) \\
& V_{n-4}(x)=\frac{(n-2 k)(n-2 k-1)(n-2 k-2)(n-2 k-3)}{4 !}-k \frac{(n-2 k)(n-2 k-1)}{2 !}+\frac{k(k-1)}{2 !} \\
& V_{n-5}(x)=\frac{\prod_{j=0}^{4}(n-2 k-j)}{5 !}-\left(\begin{array}{c}
k \\
1
\end{array}\right) \frac{\prod_{j=0}^{2}(n-2 k-j)}{3 !}+\left(\begin{array}{c}
k \\
2
\end{array}\right) \frac{\prod_{j=0}^{0}(n-2 k-j)}{1 !}, \\
& V_{n-6}(x)=V_{0}(x)=\frac{\prod_{j=0}^{5}(n-2 k-j)}{6 !}-\left(\begin{array}{c}
k \\
1
\end{array}\right) \frac{\prod_{j=0}^{3}(n-2 k-j)}{4 !}+\left(\begin{array}{c}
k \\
2
\end{array}\right) \frac{\prod_{j=0}^{1}(n-2 k-j)}{1 !}-\left(\begin{array}{c}
k \\
3
\end{array}\right) .
\end{aligned}
$$

We introduce the notation

$$
z=n-2 k, \quad z^{[0]}=1, \quad z^{[n]}=\prod_{j=0}^{n-1}(z-j) .
$$


We see for $n=6$ and $j \in[1, n]$ that

$$
V_{n-j}(x)=\frac{z^{[j]}}{j !}-\left(\begin{array}{l}
k \\
1
\end{array}\right) \frac{z^{[j-2]}}{(j-2) !}+\left(\begin{array}{l}
k \\
2
\end{array}\right) \frac{z^{[j-4]}}{(j-4) !}-\cdots+(-1)^{k}\left(\begin{array}{l}
k \\
k
\end{array}\right) \frac{z^{[j-2 k]}}{(j-2 k) !} .
$$

Summing all of the equations of Eq. (16b) for $n=6$ yields

$$
\sum_{j=0}^{n} V_{j}(x)=\sum_{j=0}^{n} \frac{z^{[j]}}{j !}-\left(\begin{array}{l}
k \\
1
\end{array}\right) \sum_{j=0}^{n-2} \frac{z^{[j]}}{j !}+\left(\begin{array}{l}
k \\
2
\end{array}\right) \sum_{j=0}^{n-2 \cdot 2} \frac{z^{[j]}}{j !}-\cdots+(-1)^{k}\left(\begin{array}{l}
k \\
k
\end{array}\right) \sum_{j=0}^{n-2 \cdot k} \frac{z^{[j]}}{j !} .
$$

Eq. (16c) was obtained for $n=6, x=-2 k / n$, and $k \in[1, n]$, but it can be easily proven by induction for arbitrary natural $n$, $x=-2 k / n$, and $k \in[1, n]$, because we see how $V_{6}(x)$ is obtained from $V_{5}(x)$. This proof is omitted for brevity.

\subsection{Proof of Eq. (10)}

Let us represent Eq. (16c) as

$$
\sum_{j=0}^{n} V_{j}(x)=\left[1-\left(\begin{array}{l}
k \\
1
\end{array}\right)+\left(\begin{array}{l}
k \\
2
\end{array}\right)-\cdots+(-1)^{k}\left(\begin{array}{l}
k \\
k
\end{array}\right)\right] \sum_{j=0}^{n-2 k} \frac{z^{[j]}}{j !}+\sum_{j=n-2 k+1}^{n} b_{j} z^{[j]},
$$

where the $b_{i}$ constants can be obtained from Eq. (16a). The first term on the right side of Eq. (16d) is equal to zero from standard results on binomial coefficients:

$$
\left[1-\left(\begin{array}{l}
k \\
1
\end{array}\right)+\left(\begin{array}{l}
k \\
2
\end{array}\right)-\cdots+(-1)^{k}\left(\begin{array}{l}
k \\
k
\end{array}\right)\right]=\left[(1-y)^{k}\right]_{y=1}=0 .
$$

The second term on the right side of Eq. (16d) is also zero, because Eq. (16a) implies that

$$
z^{[i]}=0 \text { for } i>n-2 k \text {. }
$$

Therefore,

$$
\sum_{j=0}^{n} V_{j}(x)=0 \text { for } k<n / 2 \text { and } x=-2 k / n .
$$

The case $k>n / 2$ is symmetric to the case $k<n / 2$, so the equation

$$
\sum_{j=0}^{n} V_{j}(x)=0 \text { for } k>n / 2 \text { and } x=-2 k / n
$$

can be proven similarly. Indeed, in this case $0 \leqslant n-k<n / 2$. Then replacing Eq. (17) with the equation

$$
\sum_{j=0}^{n} V_{j}(x)=\left[1-\left(\begin{array}{c}
n-k \\
1
\end{array}\right)+\left(\begin{array}{c}
n-k \\
2
\end{array}\right)-\cdots+(-1)^{n-k}\left(\begin{array}{c}
n-k \\
n-k
\end{array}\right)\right] \sum_{i=0}^{2 k-n} \frac{z^{[i]}}{i !}+\sum_{i>2 k-n} b_{i} z^{[i]}
$$

and changing Eqs. (18) and (19) in the obvious way, we obtain Eq. (19). The case $k=n / 2$ and $x=-2 k / n=-1$ is simple to prove. We combine these results to obtain

$$
\sum_{j=0}^{n} V_{j}(x)=0 \text { for } k \in[1, n] \text { and } x=-2 k / n
$$

which is identical to Eq. (10). This equation shows that the nonzero $x$ case in Eq. (5) is satisfied for $x=-2 k / n$, where $k \in[1, n]$, which proves Eq. (2).

\section{Application to biogeography}

Fig. 1 illustrates a model of species abundance in a biological habitat [12]. The emigration rate $\mu$ and the immigration rate $\lambda$ are functions of the number of species in the habitat.

The greatest possible immigration rate is $I$, which occurs when there are no species in the habitat. As the number of species increases, the habitat becomes more crowded, fewer species are able to survive immigration to the habitat, and so the immigration rate decreases. The largest number of species that the habitat can support is $S_{\max }$, at which point the immigration rate is zero.

Fig. 1 also shows that if there are no species in the habitat, then the emigration rate is zero. As the number of species increases, the habitat becomes more populated and the emigration rate increases. The maximum emigration rate is $E$, which occurs when the habitat is completely saturated with species. 


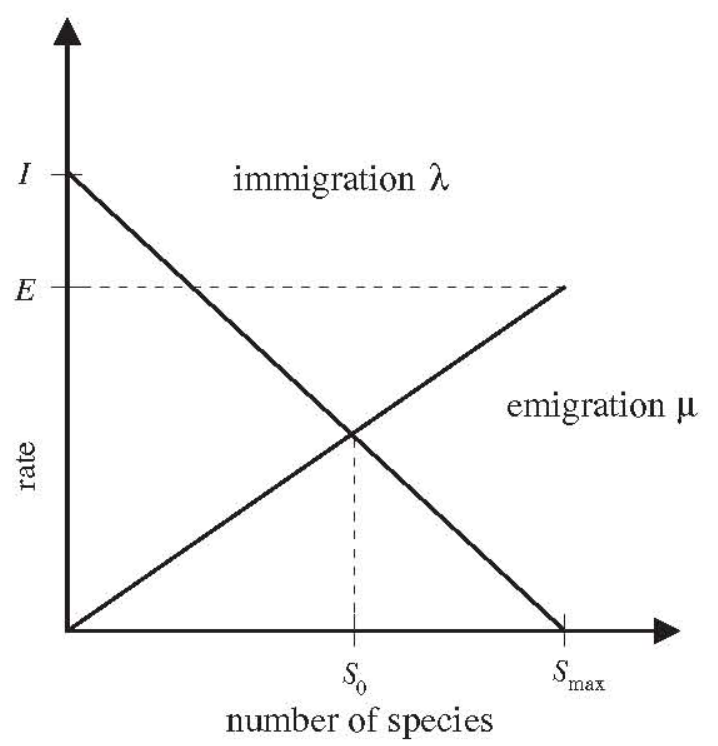

Fig. 1. Linear model of migration rates as a function of species counts.

The equilibrium number of species is $S_{0}$, at which point the immigration and emigration rates are equal. However, there will be excursions from the equilibrium species count due to transient, stochastic effects. Positive excursions from $S_{0}$ could be due to a sudden increase in immigration because of an unusually large piece of flotsam arriving from a neighboring habitat, or the extinction of a predator. Negative excursions from $S_{0}$ could be due to disease, the introduction of a new predator, or a natural catastrophe. It can take many years for species counts to reach equilibrium after such perturbations $[8,9]$.

We have shown the immigration and emigration curves in Fig. 1 as straight lines, but in general they might be nonlinear [12]. Nevertheless, the linear model provides a general description of immigration and emigration.

Now consider the probability $P_{s}$ that the habitat contains exactly $S$ species. In order for the habitat to have $S$ species at time $(t+\Delta t)$, one of the following conditions must hold:

- There were $S$ species at time $t$, and no immigration or emigration occurred between $t$ and $(t+\Delta t)$; or,

- There were $(S-1)$ species at time $t$, and one species immigrated; or,

- There were $(S+1)$ species at time $t$, and one species emigrated.

We assume that $\Delta t$ is small enough so that there is essentially zero probability of more than one immigration or emigration during a $\Delta t$ time period. Based on this reasoning, $P_{s}$ changes from time $t$ to time $(t+\Delta t)$ as follows:

$$
P_{s}(t+\Delta t)=P_{s}(t)\left(1-\lambda_{s} \Delta t-\mu_{s} \Delta t\right)+P_{s-1} \lambda_{s-1} \Delta t+P_{s+1} \mu_{s+1} \Delta t
$$

where $\lambda_{s}$ and $\mu_{s}$ are the immigration and emigration rates when there are $S$ species in the habitat.

Taking the limit of Eq. (22) as $\Delta t \rightarrow 0$ gives

$$
\dot{P}_{s}=\left\{\begin{array}{lll}
-\left(\lambda_{s}+\mu_{s}\right) P_{s}+\mu_{s+1} P_{s+1} & \text { if } & S=0, \\
-\left(\lambda_{s}+\mu_{s}\right) P_{s}+\lambda_{s-1} P_{s-1}+\mu_{s+1} P_{s+1} & \text { if } & 1 \leqslant S \leqslant S_{\max }-1, \\
-\left(\lambda_{s}+\mu_{s}\right) P_{s}+\lambda_{s-1} P_{s-1} & \text { if } & S=S_{\max } .
\end{array}\right.
$$

We define $n=S_{\max }$, and $P=\left[P_{0} \ldots P_{n}\right]^{T}$, and combine the $(n+1)$ equations of Eq. (23) to obtain

$$
\dot{P}=A_{0} P
$$

$$
\text { where } A_{0}=\left[\begin{array}{ccccc}
-\left(\lambda_{0}+\mu_{0}\right) & \mu_{1} & 0 & \cdots & 0 \\
\lambda_{0} & -\left(\lambda_{1}+\mu_{1}\right) & \mu_{2} & \ddots & \vdots \\
\vdots & \ddots & \ddots & \ddots & \vdots \\
\vdots & \ddots & \lambda_{n-2} & -\left(\lambda_{n-1}+\mu_{n-1}\right) & \mu_{n} \\
0 & \cdots & 0 & \lambda_{n-1} & -\left(\lambda_{n}+\mu_{n}\right)
\end{array}\right] \text {. }
$$


For the linear migration rates shown in Fig. 1 we have

$$
\begin{aligned}
& \mu_{s}=E S / n, \\
& \lambda_{s}=I(1-S / n) .
\end{aligned}
$$

Now consider the special case $E=I$. In this case Eq. (25) shows that

$$
\lambda_{\mathrm{S}}+\mu_{\mathrm{S}}=E=I
$$

and the $A_{0}$ matrix of Eq. (24) becomes

$$
\begin{aligned}
& A_{0}=E A \\
& \text { where } A=\left[\begin{array}{ccccc}
-1 & 1 / n & 0 & \cdots & 0 \\
n / n & -1 & 2 / n & \ddots & \vdots \\
\vdots & \ddots & \ddots & \ddots & \vdots \\
\vdots & \ddots & 2 / n & -1 & n / n \\
0 & \cdots & 0 & 1 / n & -1
\end{array}\right] \text {. }
\end{aligned}
$$

This is the same $A$ matrix as that given in Eq. (1). Based on the results of Sections 1-4, we can therefore state the following theorem, which was conjectured in [14].

Theorem 1a. The $(n+1)$ eigenvalues of the biogeography matrix $A$ are $\{0,-2 / n,-4 / n, \ldots,-2\}$.

Since $\dot{P}=0$ in steady state, since 0 is an eigenvalue of $A$, and since $\dot{P}=E A P$, the steady-state probability of each species count is given by the eigenvector which corresponds to the zero eigenvalue. This was calculated in Eq. (9) and in [14], and is repeated here in terms of the steady-state probabilities:

$$
\operatorname{Prob}(S=k)=\frac{n !}{k !(n-k) !} \text { for } k \in[0, n] \text {. }
$$

\section{Acknowledgments}

The comments of the anonymous reviewers were helpful in improving this paper from its original version. The preliminary work of Brent Gardner (Cleveland State University and the NASA Glenn Research Center) on this problem is gratefully acknowledged. This material is based on work supported by the National Science Foundation under Grant No. 0826124.

\section{References}

[1] A. Behn, K. Driessel, I. Hentsel, The eigen-problem for some special near-Toeplitz centro-skew tridiagonal matrices, arXiv:1101.5788, vol. 1 [math.SP], 2011.

[2] H.-W. Chang, S.-E. Liu, R. Burridge, Exact eigensystems for some matrices arising from discretizations, Linear Algebra and its Applications 430 (2009) $999-1006$.

[3] J. Cuminato, S. McKee, A note on the eigenvalues of special class of matrices, Journal of Computational and Applied Mathematics 234 (2010) 27242731.

[4] C. da Fonseca, On the eigenvalues of some tridiagonal matrices, Journal of Computational and Applied Mathematics 200 (2007) $283-286$.

[5] J. Elliot, The characteristic roots of certain real symmetric matrices, Master's thesis, University of Tennessee, 1953.

[6] W. Grassmann, J. Tavakoli, Spectrum of certain tridiagonal matrices when their dimension goes to infinity, Linear Algebra and its Applications 431 (2009) 1208-1217.

[7] R. Gregory, D. Carney, A Collection of Matrices for Testing Computational Algorithms, Wiley Interscience, 1969.

[8] I. Hanski, M. Gilpin, Metapopulation Biology, Academic Press, 1997.

[9] A. Hastings, K. Higgins, Persistence of transients in spatially structured models, Science 263 (1994) 1133-1136.

[10] S. Kouachi, Eigenvalues and eigenvectors of tridiagonal matrices, Electronic Journal of Linear Algebra 15 (2006) 115.

[11] S. Kouachi, Eigenvalues and eigenvectors of some tridiagonal matrices with non-constant diagonal entries, Applications Mathematicae 35 (2008) $107-$ 120.

[12] R. MacArthur, E. Wilson, The Theory of Biogeography, Princeton University Press, 1967.

[13] O. Rojo, M. Robbiano, An explicit formula for eigenvalues of Bethe trees and upper bounds on the largest eigenvalue of any tree, Linear Algebra and its Applications 427 (2007) 138-150.

[14] D. Simon, Biogeography-based optimization, IEEE Transactions on Evolutionary Computation 12 (6) (2008) $702-713$.

[15] A. Willems, Analytic results for the eigenvalues of certain tridiagonal matrices, SIAM Journal on Matrix Analysis and Applications 30 (2) (2008) 639656.

[16] Y.-C. Yueh, Eigenvalues of several tridiagonal matrices, Applied Mathematics E-Notes 5 (2005) 66-74. 